
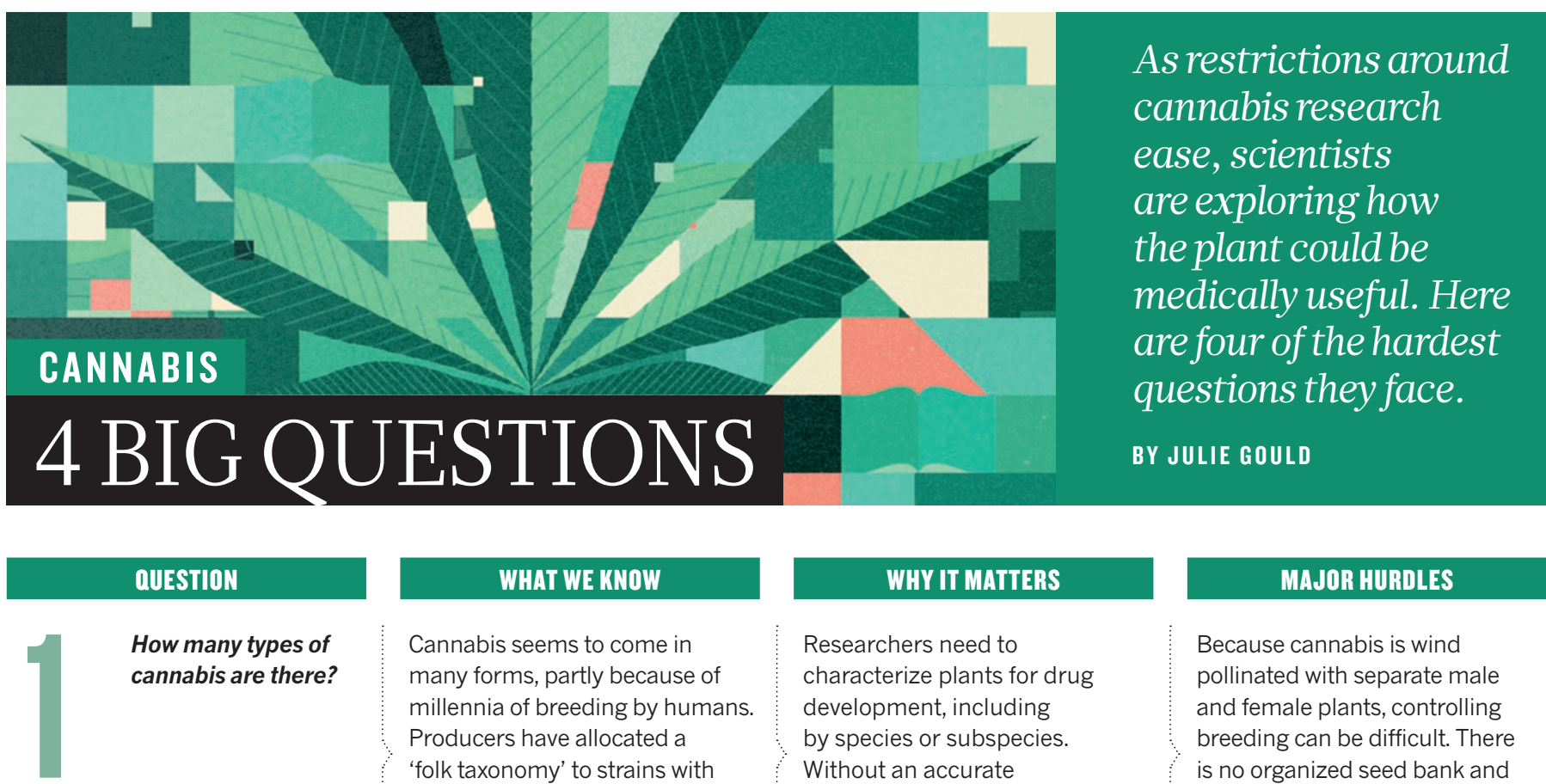

QUESTION

How many types of cannabis are there?

\section{WHAT WE KNOW}

Cannabis seems to come in many forms, partly because of millennia of breeding by humans. Producers have allocated a 'folk taxonomy' to strains with various medicinal and chemical properties, but the evolutionary relationship between species is unclear (see page S4).
WHY IT MATTERS

Researchers need to characterize plants for drug development, including by species or subspecies. Without an accurate taxonomy, there is no comparative base to work from and some varieties might become extinct.

\section{MAJOR HURDLES}

Because cannabis is wind pollinated with separate male and female plants, controlling breeding can be difficult. There is no organized seed bank and legal restrictions impede the collection of samples. Little government funding means there are few research labs.
What are the medically useful compounds in cannabis and what diseases can they treat?
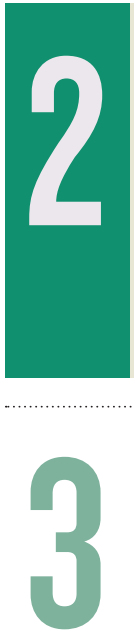

Can the endocannabinoid system be targeted by compounds other than those found in cannabis?
Of the 100 or so cannabinoids, tetrahydrocannabinol (THC) and cannabidiol have been the focus of most research. Compounds such as the volatile terpenes also seem to have intriguing properties. All constituents might act alone or in combination.
Those who use medical marijuana claim that the herb can treat a range of ailments, but evidence is thin. To test these claims and create a pharmaceutical product, the active compounds need to be isolated and investigated.
Research has been undermined by a lingering assumption that all cannabinoids share the properties of $\mathrm{THC}$ - and regulators apply the same restrictions to all of them (see page S6).

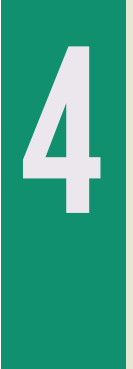

\section{What are the best ways to deliver cannabis-based medicines?}

So far, drugs that target the endocannabinoid system have failed. Cannabidiol has an anti-schizophrenia effect by increasing levels of the endocannabinoid anandamide, a type of neurotransmitter, suggesting that anandamide is the active molecule.

Delivery through the lung by smoking or vaporizing facilitates fast absorption. Administration that involves compounds passing through the liver is tricky because this organ destroys cannabinoids. Only an oral, under-tongue spray has so far been approved.
Therapies based on endogenous molecules are a mainstay of medicine. The hormone insulin was successfully given to patients with diabetes shortly after its discovery. And cortisone-based medicines can be used to relieve pain and reduce swelling.

Route of delivery determines how much of a compound is absorbed by different parts of the body, and how quickly. The faster that THC-containing medicines are delivered to the brain, the more potential there is for psychoactive side effects, as well as for abuse.
Endocannabinoids have localized actions and short halflives, so their use as systemic therapies is challenging. Endocannabinoid receptors are found throughout the body, making it difficult to target specific physiological processes without side effects.

Julie Gould is editor of NatureJobs in London.

Regulators require that cannabis-based drugs have no psychoactive effects, so delivery of THC to the brain needs to be controlled. Little is known about the metabolism of other cannabinoids and hence appropriate delivery routes. 